\title{
Simulation and Optimization of a Carbon Nanotube Electron Source
}

\author{
Alexandr Knápek, Tomáš Radlička and Stanislav Krátký \\ Institute of Scientific Instruments of the ASCR, v.v.i., Královopolská 147, 61264 Brno, Czech \\ Republic.
}

\begin{abstract}
This paper deals with an optimization of a field-emission structure concept based on vertically aligned carbon nanotubes (CNT). A design concept for a fabrication method for a gate structure based on electron beam lithography is reviewed in the first part of the paper. A single carbon nanotube is grown by the PECVD method inside the gate structure. Calculations and simulations that help determine gate structure proportions in order to obtain the best possible electron reduced brightness and to predict the cathode's electric behavior are also essential parts of this study.
\end{abstract}

\section{Introduction}

Carbon nanotubes (CNT) are a quasi one-dimensional, non-metal structure meeting many of the requirements of field-emission (FE) applications that are unavailable in traditional emitter materials [1]. It is anticipated that a carbon nanotube electron source could perhaps replace the Schottky emitter for devices utilizing a focused electron beam, such as high-resolution scanning electron microscopes (SEMs) and scanning transmission electron microscopes (STEMs). Even though the field emission from carbon nanotubes was first reported in 1995 by Rinzler's group [2] and many concepts of CNT electron sources have been proposed in recent years $[3,4]$, there is still no field emitter commercially available for utilization in electron microscopy. CNTs have several advantages over field-emission sources made from sharp metal tips.

Firstly, since the CNT is not a metal but a highly ordered crystalline structure built by a covalent $\mathrm{sp}^{2}$ (sigma) bond, the threshold for the removal of one atom is $17 \mathrm{eV}$, which is much higher than the activation energy for surface migration of a tungsten atom (3.2 eV), making the CNT much less sensitive for surface migration of the carbon atoms [5]. Additionally, carbon nanotubes have a larger Young's modulus, very high tensile strength and chemical inertness reacting only under extreme conditions or at high temperatures in the presence of oxygen or hydrogen. Moreover, carbon has a very low sputter coefficient so the current emitted from a CNT is considered stable compared with metal emitters that suffer from current fluctuations caused by the surface migration of atoms under a high electric field and by ion back sputtering. These properties make CNTs suitable candidates for an electron source [5]. Among the main advantageous parameters that could be achieved by a CNT-based emitter, the most important are very low energy width, typically $0.25 \mathrm{eV}$, high reduced brightness (up to $3 \times 10^{9}$ $\mathrm{A} / \mathrm{m}^{2} \mathrm{sr} \mathrm{V}$ ) and beam stability.

The evaluated system is based on a triode configuration incorporating an extractor electrode allowing the field strength on the tip to be easily changed. The extraction voltage can be adjusted within a range from zero to approx. $1 \mathrm{kV}$. The acceleration voltage can be set to as high as $5 \mathrm{kV}$. The radius of the CNT cathode is influenced mainly by the size of the iron particle from which it grows. The bore in the $\mathrm{SiO}_{2}$ substrate bounds the region where the catalytic iron particles react during the plasmatic growth of the CNT. The length of the tube, determined by the PECVD process, will be higher than $0.5 \mu \mathrm{m}$ so as to achieve a strong field at the tip of an order of magnitude of units of $\mathrm{V} / \mathrm{nm}$ which is necessary for the field emission. As the molybdenum used for the extractor electrode has good chemical resistivity [6], it 
is possible to functionalize the MWCNT's surface using plasmatic or chemical methods. The bore diameter in the extractor is discussed further in the text based on a method published by Radlicka [7].

Techniques capable of very high resolution and precision are needed to create such small structures. Electron beam lithography and reactive ion etching (RIE) are more than suitable for this task $[8,9]$. The described system, or more precisely the gate structure, is prepared using electron beam lithography by incorporating the following fifteen technological steps: firstly, a $300 \mathrm{~nm}$ layer of $\mathrm{SiO}_{2}$ is evaporated in a vacuum onto the surface of a silicon wafer of a (100) orientation. Secondly, a molybdenum layer that is to be used as the extractor is magnetron-sputtered on the oxide layer. The next step incorporates a 300 nm layer of Poly (methyl methacrylate), abbreviated to PMMA, using the spin-coating technique. As the PMMA layer is prepared, it is possible to exposure a pattern using a $100 \mathrm{keV}$ e-beam writer. After the chemical development of the exposed pattern, the plasma developing of resist residues is performed by oxygen plasma. The molybdenum electrode is then etched by a mixture of $\mathrm{SF}_{6}$ and $\mathrm{O}_{2}$, followed by removal of the resist layer. At this moment, it is possible to etch the $\mathrm{SiO}_{2}$ layer in order to prepare a space for CNT cathode growth. This is done using reactive ion etching with a mixture of $\mathrm{CF}_{4}$ and $\mathrm{CHF}_{3}$. The usual lithography steps are performed to prepare the layer of catalytic iron particles: Spin-coating of PMMA resist, E-beam exposure of the pattern and its chemical developing by an alcohol-based developer, followed by plasma developing of resist residues using oxygen plasma.

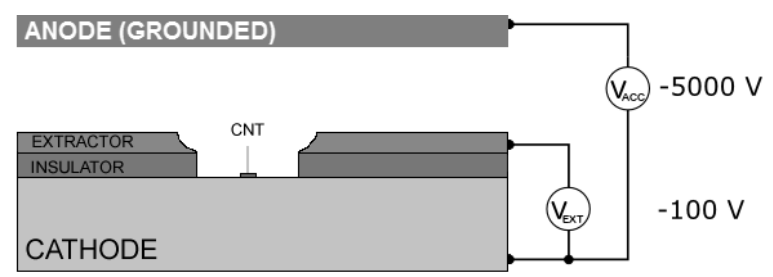

Figure 1. Fabricated gate structure after the PECVD process including an example of wiring.

A thin layer of catalytic particles is sputtered after the residues are developed, followed by the lift-off of the PMMA and the PECVD process used for CNT growth (see Figure 1).

\section{Optical properties of CNT sources}

The CNT source works primarily at room temperature and a high field, however, the setup contains an Argon laser beam, operating at $\lambda=514 \mathrm{~nm}$, which is able to increase the temperature of the CNT. For this reason we used the general thermal-field emission model [7] which is a generalization of the standard cold field emission model (or Fowler-Nordheim model). The emission current density can be calculated from the formula

$$
j_{T F}=\frac{4 \pi m e}{h^{3}} d^{2} \exp \left(-\frac{0.6 W}{d}\right) \frac{\pi p}{\sin (\pi p)}
$$

with $m$ and $e$ being the mass and the charge of the electron, respectively, and $h$ being the Planck constant. The work function for carbon $W=4.8 \mathrm{eV}$ was used. The coefficient $d$ is proportional to the field $F$ on the cathode

$$
d=\frac{e \hbar F}{2 t\left(y_{0}\right) \sqrt{2 m W}}
$$


where $\hbar$ is reduced Planck constant, $t\left(y_{0}\right)=1+0.1107 y_{0}^{1.33}$, and $y_{0}=\Delta W / W$ with $\Delta W=$ $\sqrt{e^{3} F / 4 \pi \epsilon_{0}}$ as the decrease of the work function in the presence of a strong field on the cathode surface. The coefficient $p=k T / d$ describes the influence of the temperature on the emission mechanism. The axial reduced brightness can be computed using the formula

$$
B_{r}=\frac{j_{T F}}{\pi d}
$$

In thermal-field emission model the energy distribution of escaping electrons through the potential barrier is given by:

$$
\rho_{E} \sim \frac{\exp (E / d)}{1+\exp \left(E / k_{b} T\right)}
$$

The presence of the coefficient $d$ in the exponent of the numerator causes that the energy distribution differs for different point on the cathode surface as a consequence of the different normal field on the cathode surface. $E$ denotes the energy of the electron in the CNT with respect to the Fermi level.

The electrostatic field in the system was computed using the first order finite element method (FEM). We used a triangular mesh which is sufficiently dense in the vicinity of the CNT with a tip diameter of $20 \mathrm{~nm}$ and which expands in other parts of the system. The field calculation took about five minutes with a mesh consisting of about a million triangular elements. The error of the potential calculation can be estimated by comparing the value of the potential in the nodal point of the sufficiently dense mesh with values in the same point of the refined mesh (each triangle is refined to three or more elements). The relative error of the potential with respect to the cathode-extractor voltage was in the order of 1e-4 for the mesh containing about 1.5 million mesh points. This accuracy is sufficiently high for the calculation of the emission properties and direct ray-tracing calculations. The typical potential and error of the potential in the vicinity of the cathode is plotted in Figure 2.
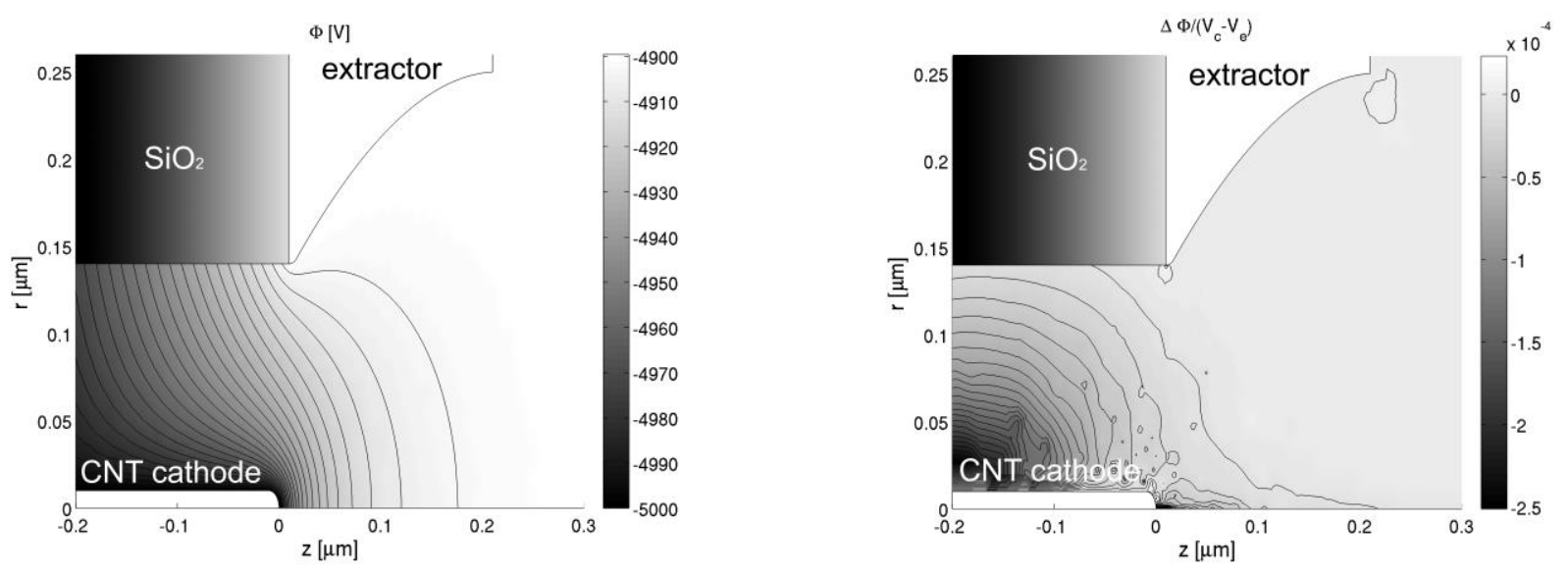

Figure 2. Potential in the vicinity of the cathode for a system with an extractor bore diameter of $0.5 \mu \mathrm{m}$ computed by the FEM of the first order and the relative error of the potential (with respect to the voltage between cathode and extractor) 
The emission was studied for several extractor bore diameters within a range from 0.5 to $2 \mu \mathrm{m}$. The field on the cathode and the emission current are determined by the extraction voltage. The emission current can be computed as a function of the extraction voltage using a thermal field emission model; see Figure 3.
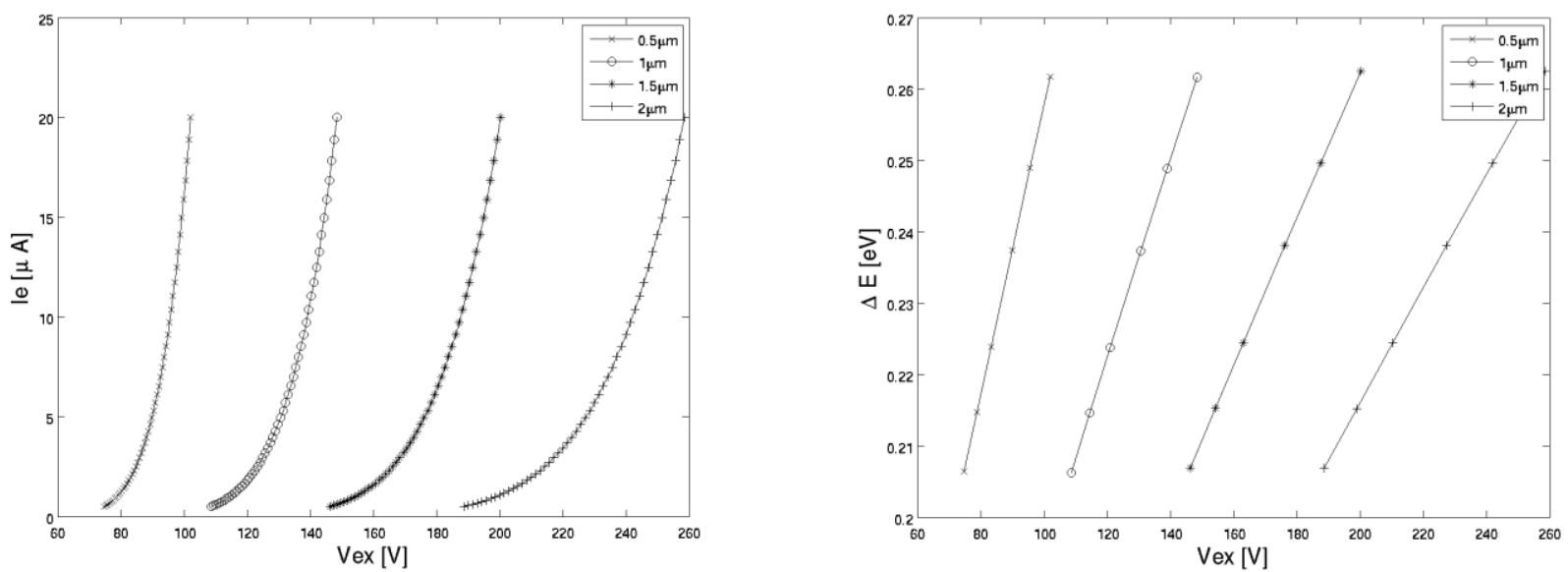

Figure 3. The emission current as a function of the extraction voltage and initial energy width of the whole beam (FW50) for several extractor bore diameters calculated by the thermal field emission model.

The bore diameter of the extractor electrode influences the dependency of the cathode field on the angle $\theta$, which is shown in Figure 4 on the left. That results in different emission current density values for different bore radii. The corresponding curves are in Figure 4 on the right. The graph on the left in Figure 5 shows the effect on the emission energy width, which is almost negligible. However, the plot on the right in Figure 5 then indicates that the effect on the axial reduced brightness must be taken into account.
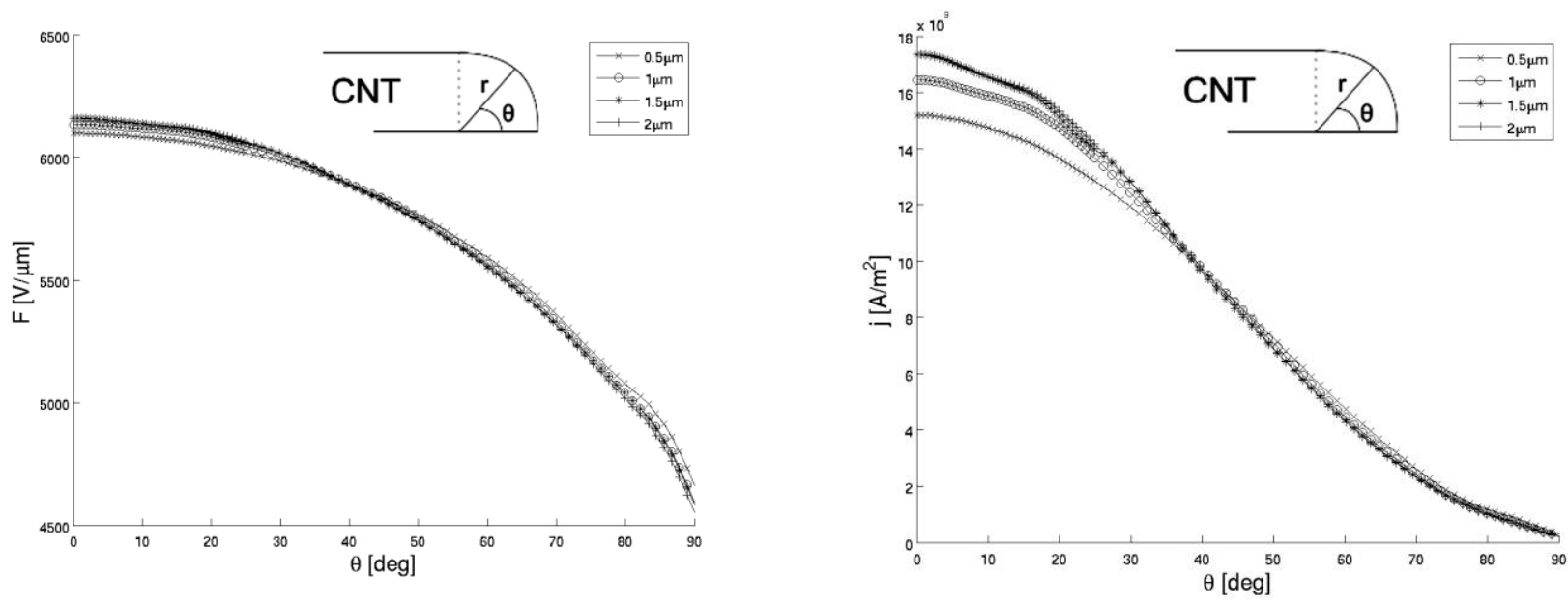

Figure 4. The field on the cathode surface (left) and the emission current density on the cathode surface for the emission current of $0.5 \mu \mathrm{A}$ and different bore radii. 
As the size of the CNT source is very small the energy distribution and brightness - the main optical parameters of the source - are strongly influenced by the stochastic Coulomb interactions (CI) in the beam. We simulated their effect on the energy distribution using a Monte-Carlo simulation presented in [7]:

a) The initial conditions of emitted electrons were randomly generated to fulfill the theoretically computed current density according to Eq. (1) and Eq. (4).

b) The electrons were traced through the system field including the effect of the stochastic CI. We simulated a beam consisting of 10,000 electrons. For high efficiency, the beam was separated into sub-beams of 400 electrons bordered by 100 electrons on both ends to eliminate the effect of beam separation [7]. The influence of the beam current on the global electrostatic field was ignored. Calculation of each beam with relative tolerance of 1e-7 took about four hours on one CPU core of an AMD FX 8350 processor running at $4.0 \mathrm{GHz}$.

c) The resulting energy width was computed from the beam properties in the anode plane.
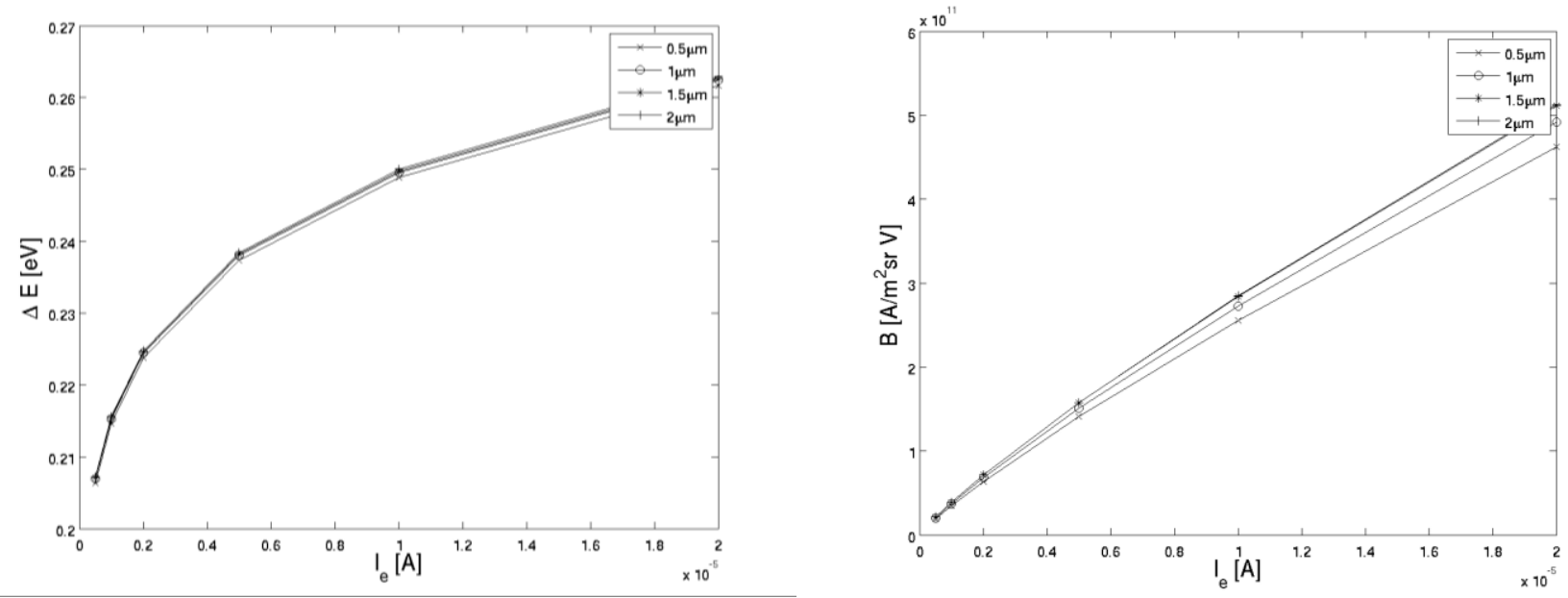

Figure 5. The emission energy width - FW50 (left) and the axial reduced brightness (right) with respect to the emission current for several bore radii.

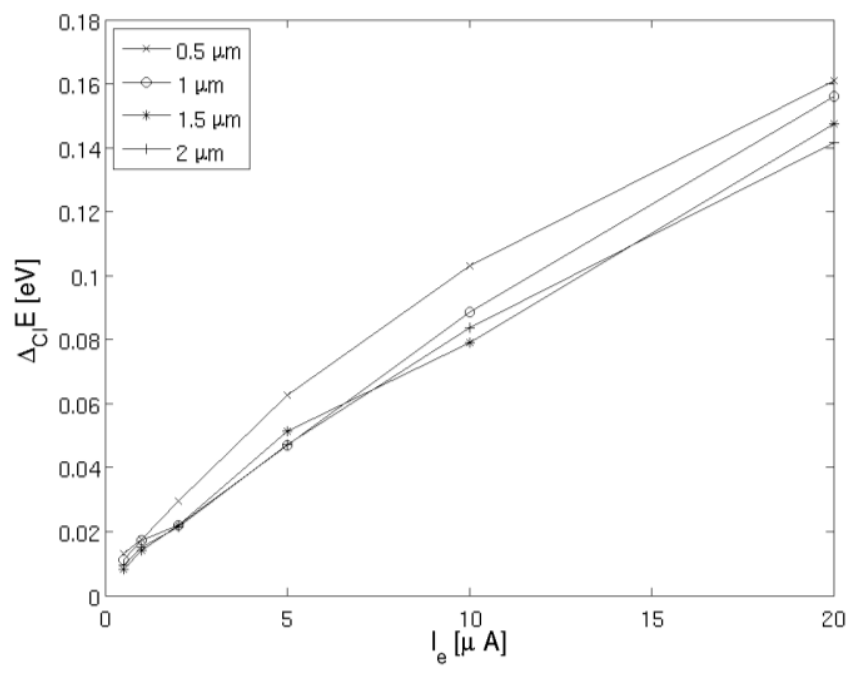

Figure 6. Increase of the energy width (FW50) due to the Boersch effect for several bore radii. 
The increase of the energy width due to the effect of the CI is presented in Figure 6. We used the FW50 characteristics, which is much simpler to determine than the FWHM. Results show an increasing effect for higher emission currents but the effect of different bore diameters seems to be within the statistical error.

\section{Conclusions}

Evaluation and optimization of the field-emission structure based on a CNT cathode is presented. The emission properties of the source were analyzed using the thermal-field emission model. The required extraction voltage was found for an emission current from 0.5 to $20 \mu \mathrm{A}$. The effect of the bore diameter in the extractor was analyzed using an MC simulation of the emitted electrons, including the effect of the stochastic CI. The effect of the CI increases the energy width from $0.01 \mathrm{eV}$ for the emission current of $0.5 \mu \mathrm{A}$ to $0.16 \mathrm{eV}$ for the highest emission current of $20 \mu \mathrm{A}$. We found no significant effect of the bore radius on the beam energy width. The effect of the Coulomb interaction on the brightness is the subject of the future research [11].

\section{References:}

[1] NS Xu and SE Huq, Mater. Sci. Eng. - R. Rep. 48 (2005), p. 47.

[2] AG Rinzler et al, Science 269 (1995), p. 1550.

[3] C Li et al, ACS Nano 6 (2012), p. 3236.

[4] Y Sun et al, Small 9 (2013), p. 3385.

[5] ND Jonge and JM Bonnard, Phil. T. R. Soc. Lond. 362 (2004), p. 2239.

[6] K Balasubramanian and M Burghard, Small 1 (2002), p. 180.

[7] T Radlicka and B Lencova, Ultramicroscopy 108 (2008), p. 445.

[8] Z Cui in "Nanofabrication Principles, Capabilities and Limits", (Springer, New York) p. 1.

[9] MA McCord and MJ Rooks in "Handbook of Microlithography, Micromachining, and

Microfabrication, Vol. 1”, ed. P Rai-Choudhury, (SPIE, Washington) p. 139.

[10] MJ Fransen et al, Adv. Imag. Electr. Phys. 111 (1999), p. 91.

[11] The authors acknowledge funding from the Ministry of Education, Youth and Sports of the Czech Republic (project number LO1212) and from Technology Agency of the Czech Republic (project number TE01020118). 